\title{
An Analytic Benchmark for the Solution to the Isotopic Fission Spectrum Mixture Problem
}

\author{
William C. Dawn \\ Department of Nuclear Engineering \\ North Carolina State University \\ Raleigh, NC, USA 27695-7909
}

January 2020

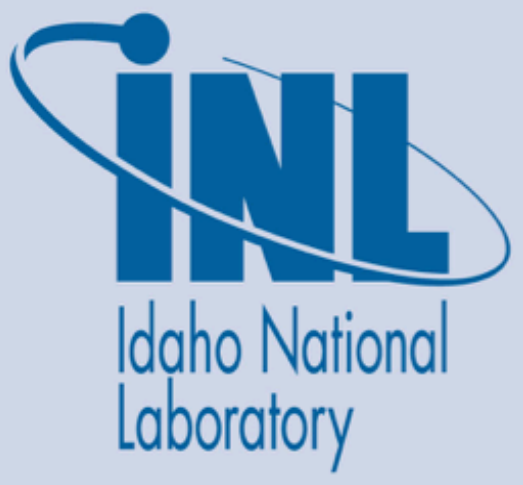

INL is a U.S. Department of Energy National Laboratory operated by Battelle Energy Alliance 


\section{DISCLAIMER}

This information was prepared as an account of work sponsored by an agency of the U.S. Government. Neither the U.S. Government nor any agency thereof, nor any of their employees, makes any warranty, expressed or implied, or assumes any legal liability or responsibility for the accuracy, completeness, or usefulness, of any information, apparatus, product, or process disclosed, or represents that its use would not infringe privately owned rights. References herein to any specific commercial product, process, or service by trade name, trade mark, manufacturer, or otherwise, does not necessarily constitute or imply its endorsement, recommendation, or favoring by the U.S. Government or any agency thereof. The views and opinions of authors expressed herein do not necessarily state or reflect those of the U.S. Government or any agency thereof.

This material is based upon work supported under an Integrated University Program Graduate Fellowship. Any opinions, findings, conclusions, or recommendations expressed in this publication are those of the author and do not necessarily reflect the views of the Department of Energy Office of Nuclear Energy. 


\title{
An Analytic Benchmark for the Solution to the Isotopic Fission Spectrum Mixture Problem
}

\author{
William C. Dawn \\ January 2020 \\ Department of Nuclear Engineering \\ North Carolina State University \\ Raleigh, NC, USA 27695-7909
}

Prepared for the

U.S. Department of Energy

Office of Nuclear Energy

Under DOE Idaho Operations Office

Contract DE-AC07-05ID14517 


\begin{abstract}
In neutron transport calculations, it is common to specify material compositions in terms of constituent isotopes. Material compositions may be described by isotope number densities and associated microscopic cross sections. For general reaction cross sections, the macroscopic cross sections of a composition are simply the summation of the sum of the products of isotope number densities and microscopic cross sections. A notable exception is the mixture of the neutron fission spectrum in fissile material.

To demonstrate proper and improper mixture of the neutron fission spectrum, a zero-dimensional test problem is developed. Using the test problem, it is demonstrated that the improper mixing of the fission spectrum results in an eigenvalue error of approximately $65 \mathrm{pcm}$. Though this may seem small, any error in such a simple calculation is unacceptable. In similar infinite-homogeneous test problems, eigenvalue errors of more than $300 \mathrm{pcm}$ have been observed. It is also shown that the error due to fission spectrum mixing can be exaggerated for computer program verification purposes.

It is concluded that the proper mixture of the neutron fission spectrum is essential for accurate neutron transport simulations. The effect of improper neutron fission spectrum mixture is demonstrated and quantified and this test problem may be used for verification purposes in the future.
\end{abstract}




\section{Contents}

1 Introduction 1

2 Zero-Dimensional, Multigroup, Isotopic Neutron Transport 2

3 Determination of the Effective Mixture Fission Spectrum 6

4 Results $\quad 7$

5 Consequences of Improper Fission Spectrum Mixture 8

6 Conclusions $\quad 10$

$\begin{array}{ll}\text { References } & 12\end{array}$

A Material Composition and Cross Sections 13 


\section{List of Tables}

1 Scalar Flux, $\phi_{g}$, in Homogeneous Mixture. . . . . . . . . . . . . . . 7

2 Fission Spectrum, $\widetilde{\chi}_{g}$, in Homogeneous Mixture. . . . . . . . . . . . . . 8

3 Perturbed Fission Spectrum, $\chi_{g}$, for ${ }^{241}$ Am for Effect Exaggeration. . . . . . 10

A.1 Composition Isotopes and Densities at $300 \mathrm{~K} . \ldots \ldots 13$

A.2 Microscopic Cross Sections for ${ }^{235} \mathrm{U}$ at $300 \mathrm{~K} \ldots \ldots \ldots$. . . . . . . . . 13

A.3 Scattering Matrix Cross Section for ${ }^{235} \mathrm{U}$ at $300 \mathrm{~K}$. . . . . . . . . . . 13

A.4 Microscopic Cross Sections for ${ }^{241}$ Am at 300 K. . . . . . . . . . . . . . . . 14

A.5 Scattering Matrix Cross Section for ${ }^{241}$ Am at 300 K. . . . . . . . . . . . . 14

A.6 Microscopic Cross Sections for ${ }^{238} \mathrm{U}$ at 300 K. . . . . . . . . . . . . . . 14

A.7 Scattering Matrix Cross Section for ${ }^{238} \mathrm{U}$ at $300 \mathrm{~K}$. . . . . . . . . . . . 14 


\section{Introduction}

Typically, material data and neutron cross sections are provided by isotope but neutron transport calculations are performed using macroscopic material properties. That is, isotope number densities are specified along with microscopic cross sections and mixing is typically performed by a computer program. This works well for typical material cross sections with two notable exceptions. First, the fission neutron production cross section, $v \Sigma_{f}$, must be mixed as a product, not merely as the fission rate, $\Sigma_{f}$. This is easily done and can subsequently be treated as any typical material property. Second, the fission spectrum, $\chi$, must be mixed and weighted with both the neutron fission production rate, $v \Sigma_{f}$, and the scalar flux, $\phi$. This poses additional challenges as the scalar flux is not known before the calculation. Therefore, the fission spectrum, $\chi$, must typically be recalculated and updated during an iterative calculation.

In this work, the mixture of an isotope-based fission spectrum is derived. It is shown that the mixture of the neutron fission spectrum requires not only number densities, but also the number of neutrons produced per fission cross section, $v \Sigma_{f}$, and the scalar flux. The dependence of the mixed fission spectrum on the scalar flux poses a significant challenge as the scalar flux is not known before the neutron transport calculation is performed. Due to this dependence, the mixed fission spectrum becomes a function of not only material composition, but also spatial position as it varies with the scalar flux. Additionally, the mixed fission spectrum must be updated in an iterative procedure as the scalar flux is calculated.

After the derivation of the mixed fission spectrum, a test problem is developed. The test problem is solved using both properly and improperly mixed fission spectra to demonstrate the importance of the method. Based on the errors observed due to improperly mixed fission spectra, conclusions are made regarding the importance of a properly mixed fission spectrum. 


\section{Zero-Dimensional, Multigroup, Isotopic Neutron Trans- port}

Consider a multigroup energy structure, indexed $g=1, \ldots, G$, such that

$$
0<E_{G}<E_{G-1}<\ldots<E_{2}<E_{1} \text {. }
$$

Then the mixture of multigroup neutron cross sections can be performed for a set of $\mathrm{N}_{\text {ISO }}$ isotopes indexed $i=1, \ldots, \mathrm{N}_{\mathrm{ISO}}$, as

$$
\begin{aligned}
\widehat{\Sigma}_{x, i, g} & =N_{i} \sigma_{x, i, g}, \\
\Sigma_{x, g} & =\sum_{i=1}^{\mathrm{N}_{\mathrm{ISO}}} \widehat{\Sigma}_{x, i, g}, \\
\Sigma_{x, g} & =\sum_{i=1}^{\mathrm{N}_{\mathrm{ISO}}} N_{i} \sigma_{x, i, g},
\end{aligned}
$$

where

$$
\begin{aligned}
N_{i}= & \text { number density for isotope } i\left[\frac{1}{\mathrm{barn} \cdot \mathrm{cm}}\right], \\
\sigma_{x, i, g}= & \text { microscopic cross section for reaction } x, \text { for isotope } i, \\
& \text { for energy group } g \text { [barn], } \\
\widehat{\Sigma}_{x, i, g}= & \text { isotope macroscopic cross section for reaction } x, \text { for isotope } i, \\
& \text { for energy group } g\left[\frac{1}{\mathrm{~cm}}\right], \\
\Sigma_{x, g}= & \text { macroscopic cross section for reaction } x, \text { for energy group } g\left[\frac{1}{\mathrm{~cm}}\right],
\end{aligned}
$$

and barn is a unit of area such that 1 barn $=10^{-24} \mathrm{~cm}^{2}$. The isotope macroscopic cross section in Eq. (2), $\widehat{\Sigma}_{x, i, g}$, is introduced for notational convenience.

Then the zero-dimensional (infinite-homogeneous) multigroup neutron transport equation can be written in terms of the constituent isotopes as

$$
\sum_{i=1}^{\mathrm{N}_{\mathrm{ISO}}} N_{i} \sigma_{t, i, g} \phi_{g}=\frac{1}{\lambda} \sum_{i=1}^{\mathrm{N}_{\mathrm{ISO}}} \chi_{i, g} \sum_{g^{\prime}=1}^{G} N_{i} v \sigma_{f, i, g^{\prime}} \phi_{g^{\prime}}+\sum_{i=1}^{\mathrm{N}_{\mathrm{ISO}}} \sum_{g^{\prime}=1}^{G} N_{i} \sigma_{s, i, g^{\prime} \rightarrow g} \phi_{g}
$$

where cross section notation is the same as previously defined and 
$\sigma_{t, i, g}=$ microscopic total cross section [barn],

$v \sigma_{f, i, g}=$ product of the number of fission neutrons and the microscopic fission cross section for energy group $g$ [barn],

$\sigma_{s, i, g^{\prime} \rightarrow g}=$ microscopic scatter cross section from energy group $g^{\prime}$ to energy group $g$ [barn],

$\phi_{g} \quad=$ scalar neutron flux for energy group $g\left[\frac{1}{\mathrm{~cm}^{2} \cdot \mathrm{s}}\right]$, and

$\lambda \quad=$ fundamental eigenvalue.

Using the cross section notations from Eq. (2) and Eq. (3), Eq. (5) can be rewritten as

$$
\Sigma_{t, g} \phi_{g}=\frac{1}{\lambda} \sum_{i=1}^{\mathrm{N}_{\text {ISO }}} \chi_{i, g} \sum_{g^{\prime}=1}^{G} \widehat{v \Sigma}_{f, i, g} \phi_{g^{\prime}}+\sum_{g^{\prime}=1}^{G} \Sigma_{s, g^{\prime} \rightarrow g} \phi_{g^{\prime}}
$$

It is useful to rewrite Eq. (6) using matrix notations. Define the following matrix quantities:

$\mathbf{S}_{t}=$ total reaction cross section matrix,

$\mathbf{S}_{s}=$ scattering matrix

$\mathbf{S}_{f}=$ fission production matrix, and

$\mathbf{X}=$ isotope fission spectrum matrix.

The dimensions and elements of the matrix quantities will be defined next. Beginning with the total and scattering macroscopic cross sections,

$$
\begin{aligned}
& \mathbf{S}_{t} \in \mathbb{R}^{G \times G}, \\
& \mathbf{S}_{t}=\operatorname{diag}\left\{\Sigma_{t, g}\right\} \\
& \mathbf{S}_{s} \in \mathbb{R}^{G \times G}, \text { and } \\
& \mathbf{S}_{s}=\left(\begin{array}{ccc}
\Sigma_{s, 1 \rightarrow 1} & \ldots & \Sigma_{s, G \rightarrow 1} \\
\vdots & \ddots & \vdots \\
\Sigma_{s, 1 \rightarrow G} & \ldots & \Sigma_{s, G \rightarrow G}
\end{array}\right)
\end{aligned}
$$

Then additional matrices for the isotope fission spectrum, $\chi_{i, g}$, and isotope fission neutron production, $\widehat{v \Sigma}_{f, i, g}$, are defined. These require additional attention to the dimension of the matrices as they are related to both the number of energy groups, $G$, and the number of 
isotopes in the composition, $\mathrm{N}_{\mathrm{ISO}}$.

$$
\begin{aligned}
\mathbf{S}_{f} & \in \mathbb{R}^{\mathrm{N}_{\mathrm{ISO}} \times G} \\
\mathbf{S}_{f} & =\left(\begin{array}{ccc}
\widehat{v \Sigma}_{f, 1,1} & \ldots & \widehat{v \Sigma}_{f, 1, G} \\
\vdots & \ddots & \vdots \\
\widehat{v \Sigma}_{f, \mathrm{~N}_{\mathrm{ISO}}, 1} & \ldots & \widehat{v \Sigma}_{f, \mathrm{~N}_{\mathrm{ISO}}, G}
\end{array}\right) \\
\mathbf{X} & \in \mathbb{R}^{\mathrm{N}_{\mathrm{ISO}} \times G} \\
\mathbf{X} & =\left(\begin{array}{ccc}
\chi_{1,1} & \ldots & \chi_{1, G} \\
\vdots & \ddots & \vdots \\
\chi_{\mathrm{N}_{\mathrm{ISO}}, 1} & \ldots & \chi_{\mathrm{N}_{\mathrm{ISO}}, G}
\end{array}\right)
\end{aligned}
$$

Finally, define a scalar flux vector of all energy groups.

$$
\begin{aligned}
& \vec{\phi} \in \mathbb{R}^{G} \\
& \vec{\phi}=\left(\begin{array}{c}
\phi_{1} \\
\vdots \\
\phi_{G}
\end{array}\right)
\end{aligned}
$$

Using these newly defined matrices and the scalar flux vector, the transport equation in Eq. (6) can be rewritten.

$$
\mathbf{S}_{t} \vec{\phi}=\frac{1}{\lambda} \mathbf{X}^{T} \mathbf{S}_{f} \vec{\phi}+\mathbf{S}_{s} \vec{\phi}
$$

Terms may then be rearranged.

$$
\begin{aligned}
\left(\mathbf{S}_{t}-\mathbf{S}_{s}\right) \vec{\phi} & =\frac{1}{\lambda} \mathbf{X}^{T} \mathbf{S}_{f} \vec{\phi} \\
\lambda \vec{\phi} & =\left(\mathbf{S}_{t}-\mathbf{S}_{s}\right)^{-1} \mathbf{X}^{T} \mathbf{S}_{f} \vec{\phi}
\end{aligned}
$$

Note that Eq. (19) is a conventional eigenvalue problem. Additionally, terms have been moved to the right-hand side although this is not traditional. The nontraditional form of Eq. (19) is necessary because the fission production term, $\left(\mathbf{X}^{T} \mathbf{S}_{f}\right)$, is singular (or nearly so) due to the low rate of fission neutron production in low-energy (thermal) groups (i.e. $\left.\chi_{i, G} \approx 0\right)$.

Eq. (19) can then be solved using a conventional eigenvalue solver such as those implemented in GNU Octave or MATLAB [1, 2]. It can be shown that Eq. (19) has a unique, maximal 
eigenvalue, $\lambda$, and an associated positive eigenvector, $\vec{\phi}[3]$. This eigenmode is known as the fundamental mode. Given material composition and cross section data, Eq. (19) can be solved for the fundamental mode. Subsequently, the scalar flux, $\vec{\phi}$, can be used to perform the appropriate determination of the fission spectrum for a given material composition. 


\section{Determination of the Effective Mixture Fission Spec- trum}

Although material composition data is typically provided in terms of constituent isotopes, it is typically desirable to calculate an effective fission spectrum for a given material composition. Using an isotope-based definition, the groupwise fission source may be written

$$
f_{g}=\sum_{i=1}^{\mathrm{N}_{\mathrm{ISO}}} \chi_{i, g} \sum_{g^{\prime}=1}^{G} \widehat{\mathrm{v}}_{f, i, g^{\prime}} \phi_{g^{\prime}}
$$

where $f_{g}$ is the groupwise fission source for energy group $g$. Equivalently, a mixed fission spectrum, $\widetilde{\chi}_{g}$, may be defined to preserve the fission source in each group. The groupwise fission source with the mixed fission spectrum has the form

$$
f_{g}=\widetilde{\chi}_{g} \sum_{g^{\prime}=1}^{G} v \Sigma_{f, g^{\prime}} \phi_{g^{\prime}}
$$

which is desirable because isotope specific information is no longer needed.

The expression for the mixed fission spectrum, $\widetilde{\chi}$, is defined by setting Eq. (20) and Eq. (21) equal. After rearranging terms, the mixed fission spectrum can be expressed.

$$
\tilde{\chi}_{g}=\frac{\sum_{i=1}^{\mathrm{N}_{\mathrm{ISO}}} \chi_{i, g} \sum_{g^{\prime}=1}^{G} \widehat{\mathrm{v \Sigma}}_{f, i, g^{\prime}} \phi_{g^{\prime}}}{\sum_{g^{\prime}=1}^{G} v \Sigma_{f, g^{\prime}} \phi_{g^{\prime}}}
$$

Note that Eq. (22) is related to the scalar flux, $\phi_{g}$. For benchmark problems, the scalar flux may be known; but this is not the case for typical neutron transport calculations. Therefore, the fission spectrum mixture in Eq. (22) generally cannot be performed a priori. Instead, the fission spectrum must be mixed repeatedly during an iterative convergence solution method. This repeated mixing of the fission spectrum is simple to implement in power iteration methods and may be easily extended to other methods [4]. For example, in a Jacobian-Free Newton-Krylov (JFNK) method the fission spectrum will now need to be mixed at every quadrature point for every residual function evaluation. Though the fission spectrum mixture must be performed during the transport calculation, it is still desirable to use the form of $\widetilde{\chi}_{g}$ because the transport solution itself does not require isotopic composition information. 
Table 1: Scalar Flux, $\phi_{g}$, in Homogeneous Mixture.

\begin{tabular}{cc}
\hline$g$ & $\phi_{g}$ \\
\hline 1 & $1.00000000000000 \times 10^{0}$ \\
2 & $2.65555181444740 \times 10^{-1}$ \\
3 & $6.35255612029976 \times 10^{-5}$ \\
4 & $1.34861637498720 \times 10^{-7}$ \\
\hline
\end{tabular}

\section{Results}

To demonstrate proper implementation of the fission spectrum mixture, a benchmark problem has been developed. An infinite-homogeneous composition of three isotopes is considered with a four-group energy structure. Cross sections are obtained using the fast reactor cross section generator, $\mathrm{MC}^{2}-3$ [5]. Microscopic cross sections and isotope densities are provided in Section A for the convenience of replicating results.

The procedure and equations from Section 2 are used to solve the eigenvalue problem and mix the fission spectrum. Briefly, the eigenvalue problem in Eq. (19) is solved to obtain the fundamental eigenvalue, $\lambda$, and the fundamental eigenvector, $\phi_{g}$. Then $\phi_{g}$ is used along with isotopic data to calculate the mixed fission spectrum, $\widetilde{\chi}_{g}$, a posteriori.

For the cross sections in this benchmark problem, it is determined that

$$
\lambda=1.02289806655878
$$

and the scalar flux and mixed fission spectrum are provided in Table 1 and Table 2 respectively. Note that the magnitude of the scalar flux is arbitrary and has been normalized such that

$$
\vec{\phi}=\frac{\vec{\phi}}{\|\vec{\phi}\|_{\infty}}
$$

where $\|\cdot\|_{\infty}$ is the infinity norm defined such that

$$
\|\vec{\phi}\|_{\infty}=\max _{g}\left|\phi_{g}\right| \quad \text { for } \quad g=1, \ldots, G .
$$


Table 2: Fission Spectrum, $\widetilde{\chi}_{g}$, in Homogeneous Mixture.

\begin{tabular}{cc}
\hline$g$ & $\widetilde{\chi}_{g}$ \\
\hline 1 & $9.85898747802817 \times 10^{-1}$ \\
2 & $1.40734497978388 \times 10^{-2}$ \\
3 & $2.78424180727868 \times 10^{-5}$ \\
4 & $5.16389731542738 \times 10^{-8}$ \\
\hline
\end{tabular}

\section{Consequences of Improper Fission Spectrum Mixture}

In some computer programs, other methods may be used to perform the mixture of the fission spectrum in an attempt to avoid the dependence on scalar flux. It is noted that these methods are inaccurate. They do not result in a correct solution to the fundamental mode.

An incorrect yet common first attempt at performing the fission spectrum mixture is to employ a weighted average with number densities. Note that the following equation is WRONG and is struck-through to avoid it being copied by others. It is included merely to demonstrate the consequences of employing this method.

$$
x_{g}=\frac{\sum_{i=1}^{\mathrm{N}_{\mathrm{ISO}}} N_{i}}{\sum_{i=1}^{\mathrm{N}_{\mathrm{ISO}}} N_{i}}
$$

Assuming that the fission spectrum, $x_{g}$, is known, the zero-dimensional transport equation may be rewritten. The fission spectrum and macroscopic fission neutron production cross sections can then be written as vectors.

$$
\begin{aligned}
& \mathbf{x} \in \mathbb{R}^{G} \\
& \mathbf{x}=\left(\begin{array}{c}
x_{1} \\
\vdots \\
x_{G}
\end{array}\right) \\
& \mathbf{n} \in \mathbb{R}^{G} \\
& \mathbf{n}=\left(\begin{array}{c}
v \Sigma_{f, 1} \\
\vdots \\
v \Sigma_{f, G}
\end{array}\right)
\end{aligned}
$$


For notational simplicity, it is useful to define a fission matrix such that $\mathbf{F}=\mathbf{x} \mathbf{n}^{T}$ (an outer product) and

$$
\mathbf{F}=\left(\begin{array}{cccc}
\chi_{1} v \Sigma_{f, 1} & \chi_{1} v \Sigma_{f, 2} & \ldots & \chi_{1} v \Sigma_{f, G} \\
\chi_{2} v \Sigma_{f, 1} & \chi_{2} v \Sigma_{f, 2} & \ldots & \chi_{2} v \Sigma_{f, G} \\
\vdots & & \ddots & \vdots \\
\chi_{G} v \Sigma_{f, 1} & \ldots & & \chi_{G} v \Sigma_{f, G}
\end{array}\right)
$$

Then the transport equation can be written as

$$
\mathbf{S}_{t} \vec{\phi}=\frac{1}{\lambda} \mathbf{F} \vec{\phi}+\mathbf{S}_{s} \vec{\phi}
$$

and subsequently rearranged into an eigenvalue problem.

$$
\lambda \vec{\phi}=\left(\mathbf{S}_{t}-\mathbf{S}_{s}\right)^{-1} \mathbf{F} \vec{\phi}
$$

As in Section 2, Eq. (32) is an eigenvalue problem in nontraditional form due to the near-singularity of the fission matrix, $\mathbf{F}$.

To demonstrate the consequences of an incorrect mixture of the fission spectrum, Eq. (32) is solved using an incorrect mixture of the fission spectrum along with the benchmark data provided in Section A. The eigenvalue result differed by $65.46 \mathrm{pcm}$ when compared to the correct solution. Though this may seem small, this is an infinite-homogeneous mixture so any error in eigenvalue greater than machine precision is unacceptable. Additionally, the effect of an incorrectly mixed fission spectrum will be exacerbated with many energy groups. For example, a similar infinite-homogeneous model employing a 33-group energy structure was observed to have over $300 \mathrm{pcm}$ error due to an incorrect fission spectrum mixture. The effect of the fission spectrum will also be exacerbated by problems with spatially dependent geometry as the fission spectrum will be spatially dependent as well due to the scalar flux dependence.

The difference of $65.46 \mathrm{pcm}$ is small but should be observable, even in single precision calculations. However, for testing or debugging purposes it may be desirable to exaggerate the difference. To cause such an exaggeration, the isotope fission spectrum, $\chi_{i, g}$, of ${ }^{241} \mathrm{Am}$ may be perturbed as given in Table 3 . Then the correct eigenvalue is $\lambda=0.92927395196420$ and the difference compared to the incorrect fission spectrum mixture is $5227.28 \mathrm{pcm}$. 
Table 3: Perturbed Fission Spectrum, $\chi_{g}$, for ${ }^{241}$ Am for Effect Exaggeration.

\begin{tabular}{cc}
\hline$g$ & $\widetilde{\chi}_{g}$ \\
\hline 1 & $5.86861 \times 10^{-1}$ \\
2 & $4.13113 \times 10^{-1}$ \\
3 & $2.62470 \times 10^{-5}$ \\
4 & $5.05900 \times 10^{-8}$ \\
\hline
\end{tabular}

\section{Conclusions}

An analytic solution to the zero-dimensional, multigroup, multiple isotope problem has been derived and solved as an eigenvalue problem. Additionally, proper calculation of the mixed fission spectrum, $\widetilde{\chi}_{g}$, has been demonstrated. Although the difference due to incorrectly mixed fission spectrum was demonstrated to be small for the chosen isotopes and four-group energy structure, the fission spectrum may be perturbed to exaggerate the effects of the fission spectrum mixture for debugging purposes. This work should be applicable as an analytic benchmark problem for the purpose of verification of computer codes. 


\section{Acknowledgements}

This work was funded in part under the Nuclear Energy Advanced Modeling and Simulation (NEAMS) program managed by the Department of Energy Office of Nuclear Energy, under DOE Idaho Operations Office Contract DE-AC07-05ID14517. Accordingly, the U.S. Government retains a nonexclusive, royalty-free license to publish or reproduce the published form of this contribution, or allow others to do so, for U.S. Government purposes. This material is also based upon work supported under an Integrated University Program Graduate Fellowship. 


\section{References}

[1] J. W. Eaton, D. Bateman, S. Hauberg, and R. Wehbring. GNU Octave Version 4.2.1 Manual: A High-Level Interactive Language for Numerical Computations. 2017.

[2] MATLAB. Version 9.6.0.1092380 (R2019a). Natick, Massachusetts: The MathWorks Inc., 2019.

[3] S. Nakamura. Computational Methods in Engineering Science. New York: Wiley, 1977.

[4] W. C. Dawn. "Simulation of Fast Reactors with the Finite Element Method and Multiphysics Models.” MA thesis. North Carolina State University, 2019.

[5] C. H. Lee and W. S. Yang. MC2-3: Multigroup Cross Section Generation Code for Fast Reactor Analysis. Tech. rep. ANL/NE-11-41. Argonne National Laboratory, 2012. 


\section{A Material Composition and Cross Sections}

All materials listed are present in the ENDF/B-VII.0 library. All cross sections are tabulated at a temperature of $300 \mathrm{~K}$ using the cross-section generator $\mathrm{MC}^{2}-3$.

Table A.1: Composition Isotopes and Densities at $300 \mathrm{~K}$.

\begin{tabular}{rrrr}
\hline Nuclide & ZAID & MC $^{2}-3$ & Isotope Density \\
\hline${ }^{235} \mathrm{U}$ & 92235 & $\mathrm{U} 235 \_7$ & 0.05 \\
${ }^{241} \mathrm{Am}$ & 95241 & AM2417 & 0.15 \\
${ }^{238} \mathrm{U}$ & 92238 & $\mathrm{U} 238 \_7$ & 0.80 \\
\hline
\end{tabular}

Table A.2: Microscopic Cross Sections for ${ }^{235} \mathrm{U}$ at $300 \mathrm{~K}$.

\begin{tabular}{rrrr}
\hline Group & $\sigma_{t, g}$ & $v \sigma_{f, g}$ & $\chi_{g}$ \\
\hline 1 & $9.06034 \times 10^{0}$ & $3.15664 \times 10^{0}$ & $9.85899 \times 10^{-1}$ \\
2 & $1.28790 \times 10^{1}$ & $4.36341 \times 10^{0}$ & $1.40733 \times 10^{-2}$ \\
3 & $2.49090 \times 10^{1}$ & $2.14413 \times 10^{1}$ & $2.76717 \times 10^{-5}$ \\
4 & $6.04504 \times 10^{1}$ & $7.61862 \times 10^{1}$ & $5.04363 \times 10^{-8}$ \\
\hline
\end{tabular}

Table A.3: Scattering Matrix Cross Section for ${ }^{235} \mathrm{U}$ at $300 \mathrm{~K}$.

\begin{tabular}{l|rrrr}
\hline & 1 & 2 & 3 & 4 \\
\hline 1 & $7.40592 \times 10^{0}$ & & & \\
2 & $1.95241 \times 10^{-1}$ & $1.05387 \times 10^{1}$ & & \\
3 & $2.24470 \times 10^{-4}$ & $3.34712 \times 10^{-4}$ & $1.18818 \times 10^{1}$ & \\
4 & $2.84816 \times 10^{-6}$ & $4.04812 \times 10^{-7}$ & $3.12386 \times 10^{-4}$ & $1.13102 \times 10^{1}$ \\
\hline
\end{tabular}


Table A.4: Microscopic Cross Sections for ${ }^{241}$ Am at $300 \mathrm{~K}$.

\begin{tabular}{rrrr}
\hline Group & $\sigma_{t, g}$ & $v \sigma_{f, g}$ & $\chi_{g}$ \\
\hline 1 & $9.52564 \times 10^{0}$ & $1.34216 \times 10^{0}$ & $9.86861 \times 10^{-1}$ \\
2 & $1.31416 \times 10^{1}$ & $4.64501 \times 10^{-2}$ & $1.31131 \times 10^{-2}$ \\
3 & $2.39526 \times 10^{1}$ & $2.01069 \times 10^{-1}$ & $2.62470 \times 10^{-5}$ \\
4 & $4.09284 \times 10^{1}$ & $6.50220 \times 10^{-1}$ & $5.05900 \times 10^{-8}$ \\
\hline
\end{tabular}

Table A.5: Scattering Matrix Cross Section for ${ }^{241} \mathrm{Am}$ at $300 \mathrm{~K}$.

\begin{tabular}{r|rrrr}
\hline & 1 & 2 & 3 & 4 \\
\hline 1 & $8.17273 \times 10^{0}$ & & & \\
2 & $2.23929 \times 10^{-1}$ & $1.11373 \times 10^{1}$ & & \\
3 & $3.37577 \times 10^{-4}$ & $2.60617 \times 10^{-4}$ & $1.26347 \times 10^{1}$ & \\
4 & $4.62453 \times 10^{-6}$ & $2.76773 \times 10^{-7}$ & $3.38661 \times 10^{-4}$ & $1.05887 \times 10^{1}$ \\
\hline
\end{tabular}

Table A.6: Microscopic Cross Sections for ${ }^{238} \mathrm{U}$ at $300 \mathrm{~K}$.

\begin{tabular}{rrrr}
\hline Group & $\sigma_{t, g}$ & $v \sigma_{f, g}$ & $\chi_{g}$ \\
\hline 1 & $9.19917 \times 10^{0}$ & $1.76275 \times 10^{-1}$ & $9.84512 \times 10^{-1}$ \\
2 & $1.22540 \times 10^{1}$ & $6.45956 \times 10^{-6}$ & $1.54573 \times 10^{-2}$ \\
3 & $1.21864 \times 10^{1}$ & $1.26832 \times 10^{-3}$ & $3.04023 \times 10^{-5}$ \\
4 & $1.19987 \times 10^{1}$ & $1.19020 \times 10^{-5}$ & $5.49910 \times 10^{-8}$ \\
\hline
\end{tabular}

Table A.7: Scattering Matrix Cross Section for ${ }^{238} \mathrm{U}$ at $300 \mathrm{~K}$.

\begin{tabular}{r|rrrr}
\hline & 1 & 2 & 3 & 4 \\
\hline 1 & $8.88152 \times 10^{0}$ & & & \\
2 & $1.47289 \times 10^{-1}$ & $1.19811 \times 10^{1}$ & & \\
3 & $3.71279 \times 10^{-5}$ & $2.92958 \times 10^{-4}$ & $1.15047 \times 10^{1}$ & \\
4 & $3.91127 \times 10^{-7}$ & $0.00000 \times 10^{0}$ & $2.53909 \times 10^{-4}$ & $9.53619 \times 10^{0}$ \\
\hline
\end{tabular}

